\title{
The frequency of occurrence of novae hosting an ONe white dwarf
}

\author{
P. Gil-Pons ${ }^{1}$, E. García-Berro ${ }^{1,2}$, J. Joséé, ${ }^{2,3}$, M. Hernanz ${ }^{2,4}$, and J. W. Truran ${ }^{5}$ \\ 1 Departament de Física Aplicada, Universitat Politècnica de Catalunya, c/Jordi Girona s/n, Mòdul B-4, Campus Nord, \\ 08034 Barcelona, Spain \\ 2 Institute for Space Studies of Catalonia, c/Gran Capità 2-4, Edif. Nexus 104, 08034 Barcelona, Spain \\ 3 Departament de Física i Enginyeria Nuclear, Universitat Politècnica de Catalunya, Av. Víctor Balaguer, s/n, 08800, \\ Vilanova i la Geltrú (Barcelona), Spain \\ ${ }^{4}$ Institut de Ciències de l'Espai (CSIC) \\ 5 Department of Astronomy and Astrophysics, 5640 S. Ellis Ave, Chicago, IL 60637, USA
}

Received 18 October 2002 / Accepted 4 June 2003

\begin{abstract}
In this paper, we revisit the problem of the determination of the frequency of occurrence of galactic nova outbursts which involve an oxygen-neon (ONe) white dwarf. The improvement with respect to previous work on the subject derives from the fact that we use the results that our evolutionary calculations provide for the final mass and for the chemical profiles of intermediate-to-massive primary components of close binary systems. In particular, the final evolutionary stages, such as the carbon burning phase, have been carefully followed for the whole range of masses of interest. The chemical profiles obtained with our evolutionary code are of interest in determining the chemical composition of the ejecta after being processed through the thermonuclear runaway, although such other factors as the efficiency of the mixing between the accreted material and that of the underlying white dwarf must also be considered. In our calculations of the frequency of occurrence of nova outbursts involving an ONe white dwarf, we also take into account the observational selection effects introduced by the different recurrence times of the outbursts and by the spatial distribution of novae. In spite of the very different evolutionary sequences, we find that approximately $1 / 3$ of the observed nova outbursts should involve an oxygen-neon white dwarf, in agreement with previous theoretical estimates.
\end{abstract}

Key words. stars: evolution - stars: binaries: general - stars: novae, cataclysmic variables - stars: white dwarfs

\section{Introduction}

The formation of massive oxygen-neon white dwarfs in cataclysmic binaries and the occurrence of ONe novae was first suggested - and its consequences investigated - by Law \& Ritter (1984), even before any observational evidence for such objects was found. Subsequently, the determination of the frequency of occurrence of ONe white dwarfs in galactic classical nova systems has also been considered by other authors see, for instance, Truran \& Livio (1986), Ritter et al. (1991), and Livio (1992). If the evolution of primary components in close binary systems (CBSs) were identical to those of single stars, and if observational selection effects were negligible (which is not the case), one would expect a peak in the mass distribution of white dwarfs around the canonical value of $0.6 M_{\odot}$ (Bergeron et al. 1992) found for single white dwarfs. We would thus expect to find a small number of oxygenneon white dwarfs in nova systems. In contrast, there is an increasing body of observational information indicating that the

Send offprint requests to: E. García-Berro, e-mail: garcia@fa.upc.es actual mass distribution of white dwarfs in galactic nova systems is skewed toward higher masses. In fact, the mass distribution for galactic nova systems is peaked around $1.0 M_{\odot}$ (Webbink 1990). Moreover, the observed abundances in the ejecta of some novae - see, e.g. Gehrz et al. (1998), for a recent review of the abundances inferred from observations of novae - show high neon enhancements (Saizar et al. 1992; Andreä et al. 1994; Austin et al. 1996; Vanlandingham et al. 1997). This again strongly suggests, when compared with theoretical models (José \& Hernanz 1998), that the underlying white dwarf is made of oxygen and neon, and therefore that it is a massive white dwarf. We should thus expect that a significant fraction of nova systems contain ONe white dwarfs.

Ritter et al. (1991) estimated that the frequency of occurrence of nova outbursts hosting an $\mathrm{ONe}$ white dwarf in the Galaxy was between $25 \%$ and $57 \%$, in good agreement with the previous work by Truran \& Livio (1986), and compatible with the available observational data. However, in a subsequent discussion of this issue in which the observational uncertainties were further scrutinized, Livio \& Truran (1994) adopted a more cautious approach to the identification of ONe novae. 
They chose to classify novae into three distinct groups. The first group corresponds to novae with high helium abundances and modest CNO nuclei enrichment. The second group includes novae with high $\mathrm{CNO}$ nuclei enrichment and moderate neon enhancement. Finally, the third group corresponds to those novae with high helium to hydrogen abundance ratio and both neon and heavy element enrichment. The well established $\mathrm{ONe}$ novae should then be the ones belonging to the third group constituting two or three objects out of 18 according to Livio \& Truran (1994) - whereas less restrictive ONe nova identifications predict that $\mathrm{ONe}$ novae constitute about one third of the sample. Whatever the appropriate fraction of ONe novae might be, it is worth noting at this point that observational selection effects also play a key role in determining this frequency (Livio \& Soker 1984; Ritter \& Burkert 1986; Ritter et al. 1991).

Another factor that has a considerable influence on the mass distribution of novae is the evolutionary history of the binary system previous to the formation of the nova. Indeed, the evolutionary history of the precursor system can influence the composition of the nova ejecta. It is clear now that all the possibilities for an in situ formation of the abundance pattern found in the ejecta of novae must be rejected for several reasons. First, the amount of CNO products observed in the ejecta of novae is much greater than can be expected to characterize the matter accreted from a companion star of solar-like composition. Second, breakout from the $\mathrm{CNO}$ cycle to produce nuclei like $\mathrm{Ne}, \mathrm{Na}$, and $\mathrm{Mg}$ cannot occur at the temperatures smaller than 300 million degrees achieved in nova thermonuclear runaways. Third, the abundance pattern of the ejecta of novae cannot be explained by a previous case BB mass-transfer episode. It then follows that the explanation of the enriched heavy element composition of the ejecta of classical novae must involve some degree of dredge-up of matter from the underlying $\mathrm{CO}$ or ONe white dwarf.

Helium and carbon-burning reactions that occur in the core of the primary during the prior evolutionary history of the white dwarf progenitor can explain the formation of the neon and the heavy elements that characterize the abundance pattern of the ejecta from ONe novae. Thus the importance of reliable composition profiles for massive white dwarfs, resulting from detailed evolutionary calculations, becomes clear. In this context, it is worth noting that, due to the lower carbon abundance in the outer layers of ONe white dwarfs, these objects are able to accrete more massive envelopes (José \& Hernanz 1998) between successive outbursts and, therefore, the degree of degeneracy reached at the bottom of the accreted layers is higher and the explosions are more violent than in $\mathrm{CO}$ white dwarfs of similar masses. The higher temperatures that $\mathrm{ONe}$ white dwarfs in novae are able to reach, together with the presence of "seed" nuclei with $A \geq 20$, have also consequences for the nature of the material processed during the outbursts: specifically, we expect that heavier nuclei can be formed in these environments. However, due to the relatively small masses of nova ejecta, and in spite of their moderately high frequency, novae do not represent a significant source of heavy element enrichment of Galactic matter. Finally it should be said that although the characteristic isotopes ejected by novae involving $\mathrm{CO}$ and $\mathrm{ONe}$ white dwarfs are similar $\left({ }^{13} \mathrm{C},{ }^{15} \mathrm{~N}\right.$, and $\left.{ }^{17} \mathrm{O}\right)$, their ejecta have distinguishing imprints: e.g. ${ }^{7} \mathrm{Li}$ is produced in greater amounts by $\mathrm{CO}$ novae while both ${ }^{22} \mathrm{Na}$ and ${ }^{26} \mathrm{Al}$ are understood to be produced by ONe novae (José \& Hernanz 1998).

The mechanism responsible for the outward mixing (dredge-up) of white dwarf core matter into the hydrogen-rich envelope remains to be clearly identified. A variety of possible mechanisms have been examined, including shear-driven turbulent mixing (Kippenhahn \& Thomas 1978), Eckman spinup (Livio \& Truran 1987), diffusion induced core convection (Prialnik \& Kovetz 1984), dredge-up driven by convective overshoot (Glasner et al. 1997), and most recently mixing by wind-driven gravity wave breaking on the surface of the white dwarf (Rosner et al. 2001; Calder et al. 2002). Although it is true that it is not yet known how much material is mixed, it is also clear that reliable initial models for the masses and compositions of the white dwarf components of nova systems, as a function of the mass of the progenitor, are required for a full understanding of the chemical composition of the ejecta from novae.

In this paper we recompute the frequency of white dwarfs with ONe cores in novae, taking into account both the observational selection effects and our recent evolutionary sequences for binary systems with intermediate-to-massive primaries (Gil-Pons \& García-Berro 2001, 2002). As will be shown below, we expect that some novae hosting massive white dwarfs (e.g., an ONe degenerate core) will show Ne enrichments while others will not, due to the presence of a relatively thick CO buffer. We also discuss the consequences of these evolutionary sequences, and in particular of this $\mathrm{CO}$ buffer, for the expected abundance pattern of the ejecta. The paper is organized as follows. In Sect. 2, we briefly comment on the standard double common envelope scenarios leading to novae that host massive white dwarfs. Section 3 presents our main results for the remnant masses and their compositions. In Sect. 4, we discuss the observational selection effects arising from both the recurrence intervals between successive nova outbursts in individual systems and the spatial distribution of novae in the Galaxy. In Sect. 5, we comment on the expected abundances of the nova ejecta. Discussion and conclusions are presented in Sect. 6.

\section{Scenarios leading to novae}

Evolutionary sequences for primaries of mass $10.0 M_{\odot}$ (Gil-Pons \& García-Berro 2001) and $9.0 M_{\odot}$ (Gil-Pons \& García-Berro 2002) have previously been computed. For the present work, we have computed additional evolutionary sequences for primaries of masses $M_{\mathrm{ZAMS}}=10.5,11.0,11.5$, and $12.0 M_{\odot}$. The physical inputs are the same as in GilPons \& García-Berro (2001) and the results are very similar to those presented there for the whole range of masses. The only significant difference with respect to our previous work occurs for the primary of $12.0 M_{\odot}$, which ignites carbon at its center (see Sect. 3 below). For binary systems hosting primaries with a mass smaller than $9.0 M_{\odot}$ we have adopted the results of Iben \& Tutukov (1985) and Iben (1990). Our systems undergo the so-called case BB mass transfer (Delgado \& Thomas 1981; de Loore \& Doom 1992). The first Roche lobe 


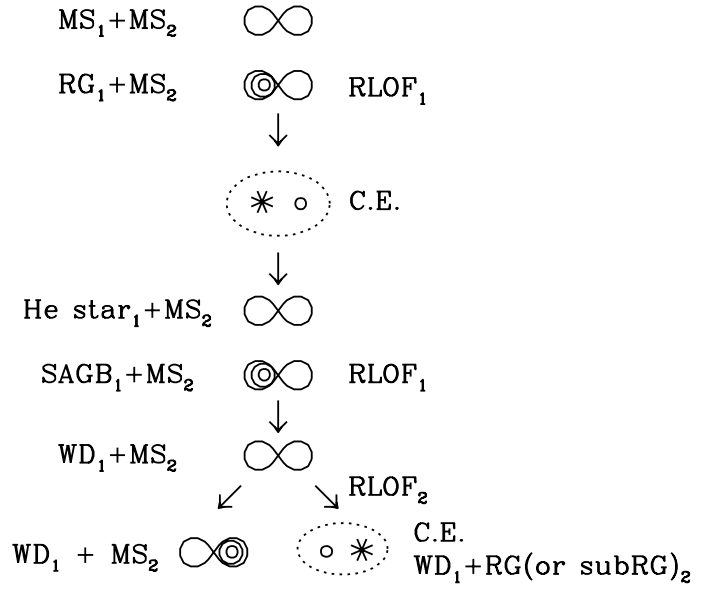

Fig. 1. Outline of pre-nova scenarios hosting massive white dwarfs. $M S, S A G B$ and WD represent, respectively, main sequence, superasymptotic giant branch star and white dwarf. RLOF and C.E. refer to Roche lobe overflow and common envelope phase, and the subindices 1 and 2 correspond to the primary and the secondary component, respectively.

overflow (RLOF) from the primary component occurs when hydrogen burning has begun in a shell and a deep convective envelope forms around the helium core. Mass loss in the presence of convection induces unstable mass transfer between the components and leads to the formation of a common envelope (Paczyński 1976). This, in turn, induces orbital shrinkage. The primary loses most of its hydrogen-rich envelope during the first RLOF, and most of the mass is lost from the system. The second RLOF episode leaves a remnant which consists of an ONe core surrounded by a CO buffer and a thin helium layer.

Roche lobe overflow from the secondary (and thus reverse mass transfer) can occur, whether due to further evolution of this star or to angular momentum losses. A nova can only occur for suitable mass transfer rates, specifically $\lesssim 10^{-8} M_{\odot} \mathrm{yr}^{-1}$, However, systems in which the mass transfer rates are larger are not necessarily prevented from evolving to novae. In particular, Politano (2002) has suggested the possibility that such systems might undergo first a violent phase during which the mass transfer rates are large, ensuing important mass and angular momentum losses from the system, and then a second phase, involving mass transfer rates low enough to allow the development of nova outbursts. Another possibility for the occurrence of nova outbursts involving a phase of high mass transfer rates has been discussed by Shara \& Prialnik (1994), and Shara (1994), who suggested the build-up of an ONe-rich layer on top of a $\mathrm{CO}$ white dwarf as a consequence of weak hydrogen and helium flashes. A subsequent phase of low mass accretion rates would then trigger a nova explosion. However, fine tuning of model parameters is necessary to obtain a realistic nova event, for the conditions proposed by these authors. It thus seems that, while we may have a good qualitative picture of the nature and evolution of the progenitors of novae, many detailed features of their evolution remain to be fully explored numerically. A schematic view of our scenario is presented in Fig. 1.

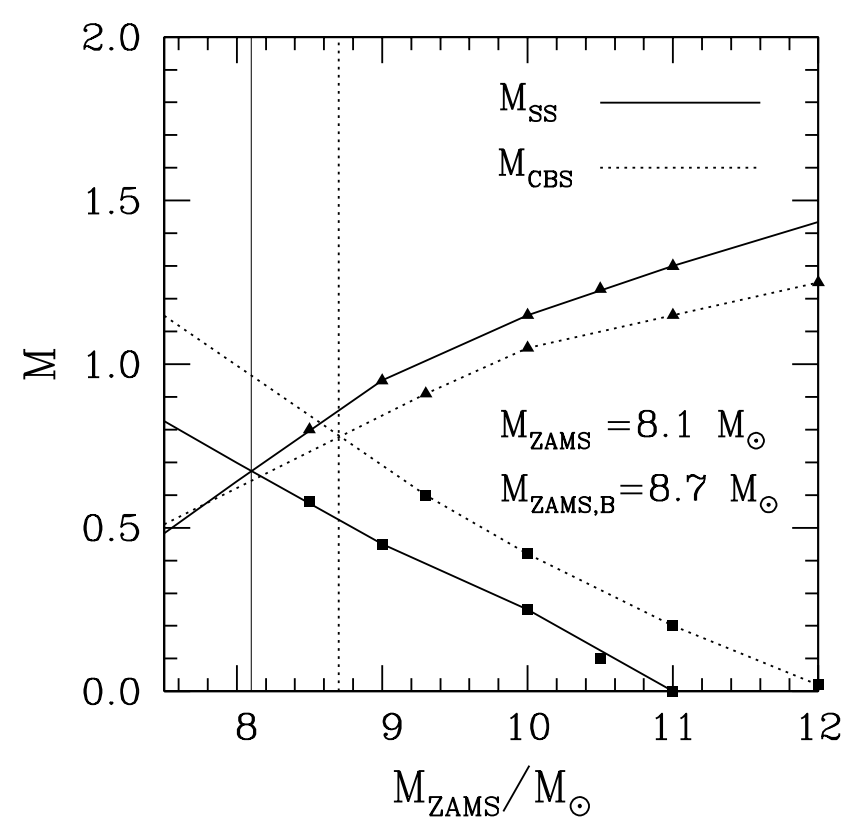

Fig. 2. Size of the $\mathrm{CO}$ cores at the beginning of carbon burning (triangles) and mass point at which carbon is ignited (squares). The results for the evolution of a single star are represented as solid lines, whereas dotted lines show the results for the evolution in a CBS. See text for additional details.

\section{The degenerate cores}

The size of the CO core just before carbon burning sets in, and the mass at which carbon ignition occurs are shown in Fig. 2, as a function of the mass on the zero age main sequence (ZAMS); these are shown both for the case of single stars (solid lines) and for stars in close binary systems (dotted lines). These cores are the result of considering a case BB non-conservative mass transfer episode between the components. The first mass transfer episode occurs when the primary star is ascending the red giant branch, and a deep convective envelope is formed. In such a scenario, a common envelope phase is very likely to occur, and significant amounts of mass and angular momentum can be lost from the system. Systems initially closer may undergo mass transfer while the donor is still surrounded by a radiative envelope. In this case, we would obtain somewhat more massive ONe white dwarfs. The differences we have found in our calculations are about $0.02 M_{\odot}$ between the ONe core resulting from the conservative and non-conservative mass transfer case, whereas the differences between considering single star evolution and evolution in a close binary are about $0.1 M_{\odot}$. Hence, for reasonable choices of the orbital parameters the size of the resulting He core is quite insensitive to the computational details (Gil-Pons \& García-Berro 2002). It follows that, even though the initial-to-final mass relation of the primary components is not unique but depends to some extent on the initial separation and mass ratio of the binary, these differences are considerably smaller than those resulting from taking into account the consequences of binary evolution.

As one can see, the remnant cores of close binary components tend to be smaller. This behavior has also been found in several other theoretical works - see, for instance, 
Table 1. Size of the final cores as a function of the ZAMS mass for binary star evolution.

\begin{tabular}{lrc}
\hline \hline$M_{\mathrm{ZAMS}}$ & $M_{\mathrm{ONe}}$ & $M_{\mathrm{ONe}+\Delta \mathrm{CO}}$ \\
\hline 9.3 & 1.00 & 1.07 \\
10.0 & 1.05 & 1.09 \\
10.5 & 1.14 & 1.15 \\
11.0 & 1.21 & 1.22 \\
11.5 & 1.30 & 1.31 \\
12.0 & 1.33 & 1.33 \\
\hline
\end{tabular}

Iben \& Tutukov (1985) and references therein. Note that for this range of masses, carbon is ignited off-center and that, as the mass of the primary increases, the mass at which carbon is ignited approaches the center. In particular, we find that for the case of isolated evolution, stars of mass $M_{\text {ZAMS }} \simeq 11 M_{\odot}$ ignite carbon at the center, whereas this limit turns out to be $M_{\text {ZAMS }} \simeq 12 M_{\odot}$ for the case of the evolution in a CBS. We also note that, as the mass of the star decreases from $12 M_{\odot}$ to $9 M_{\odot}$, the mass of the degenerate $\mathrm{CO}$ core correspondingly decreases, whereas the point at which carbon is ignited approaches the He-C discontinuity. The intersection of the two curves determines the minimum mass required for carbon ignition to take place. The lines intersect at $M_{\mathrm{ZAMS}} \simeq 8.1 M_{\odot}$ and $8.7 M_{\odot}$, respectively. This represents the lower mass limit for the formation of ONe white dwarfs. However, this mass cannot be taken as the limiting mass separating $\mathrm{CO}$ from $\mathrm{ONe}$ white dwarfs, since the fact that carbon ignition occurs does not necessarily imply that burning is going to be extended enough in the $\mathrm{CO}$ core to change drastically its composition and form an ONe white dwarf. In fact we find that the limiting masses are somewhat larger, $9.0 M_{\odot}$ and $9.3 M_{\odot}$ respectively and that for masses smaller than these the carbon burning reactions never proceed at a significant rate. Note, however, that in any case we always obtain smaller cores for the evolution in CBSs. This result influences the expected frequency of ONe white dwarfs, since the larger value of the minimum mass allowing for the formation of novae hosting an $\mathrm{ONe}$ white dwarf obtained here, combined with the fact that IMF tends to favour the formation of low-mass stars, will also favour the formation of $\mathrm{CO}$ novae. The effects of binary evolution, even though they may be weaker than the observational selection effects, will thus act against novae hosting an ONe white dwarf.

Another factor which influences the expected frequency of nova systems hosting an $\mathrm{ONe}$ white dwarf is the relationship between the initial and final mass. The degenerate cores obtained in our simulations have an ONe core surrounded by a $\mathrm{CO}$ buffer. Figure 3 shows the relationship between the initial mass of the primary and the mass of the white dwarf (solid line), and the size of the ONe core (dotted line) for both the single and the binary cases. For the sake of completeness, these two quantities are also presented in Table 1.

With respect to the final chemical composition profiles obtained in our evolutionary sequences after carbon has been exhausted in the central regions, the most important characteristic of the degenerate cores is the existence of two different regions: an inner core where oxygen and neon are the

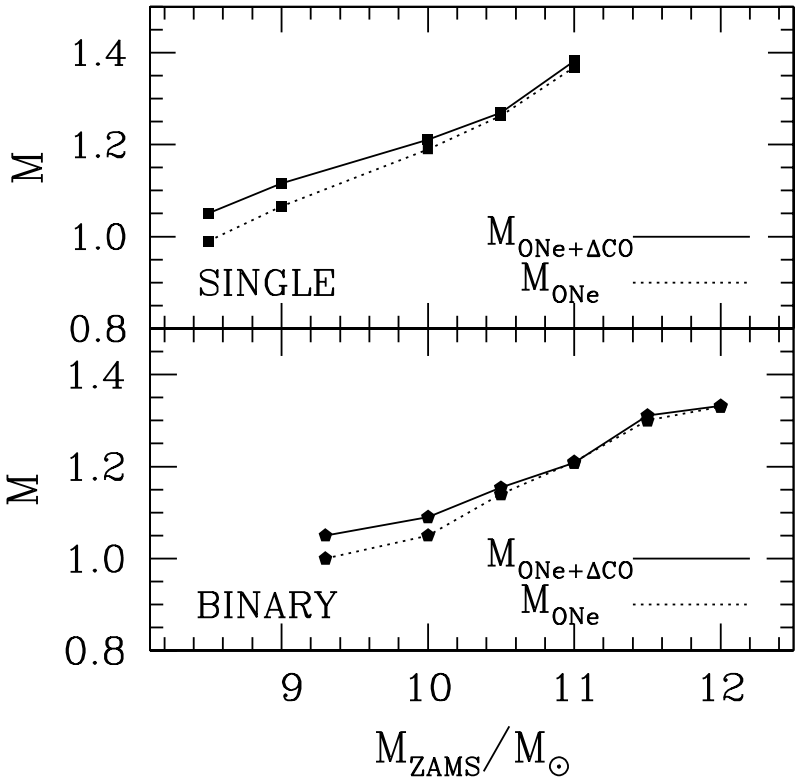

Fig. 3. Size of the final cores as a function of the ZAMS mass for single and binary star evolution.

dominant constituents, and a carbon-oxygen layer surrounding this core, where extensive helium burning has occurred, but where the physical conditions are not compatible with carbon burning. As one can see in Table 1, the size of this CO layer decreases as the total white dwarf mass increases, from $\Delta M_{\mathrm{CO}}=0.07 M_{\odot}$ for the remnant of the $9.3 M_{\odot}$ star, down to $\Delta M_{\mathrm{CO}}<0.01 M_{\odot}$, for the remnant of the $12 M_{\odot}$ primary. The presence of this $\mathrm{CO}$ buffer has two foreseeable consequences for the nova outbursts. First, one can expect that the first nova outbursts occurring on such a core will not show substantial neon enrichments. Depending upon the efficiency of the mixing between the material of the inner core and of the accreted material, neon might not be detectable until most of the CO buffer is dredged-up and ejected. Also, the presence of an important amount of carbon increases the rate of energy generation by the $\mathrm{CNO}$ cycles at lower temperatures and thus ensures an earlier occurrence of thermonuclear runaway. A "naked" ONe white dwarf would be able to accrete more mass between outbursts and would allow for more massive ejecta (José et al. 2003).

\section{The observed rates of oxygen-neon nova outbursts}

The observed fraction of nova outbursts in which an ONe white dwarf is involved depends upon the relative frequency of $\mathrm{ONe}$ white dwarfs in close binary systems and upon several selection effects. We will consider here selection effects of two types. First we will take into account those related to the recurrence time between outbursts. Since massive white dwarfs - and, hence, ONe white dwarfs - experience nova outbursts more frequently, there will be a clear selection bias favouring their detection. Second, more massive white dwarfs produce more luminous outbursts, thus favouring their detection as well.

In deriving the frequency of nova outbursts in which an ONe white dwarf is involved, we follow closely the treatments 


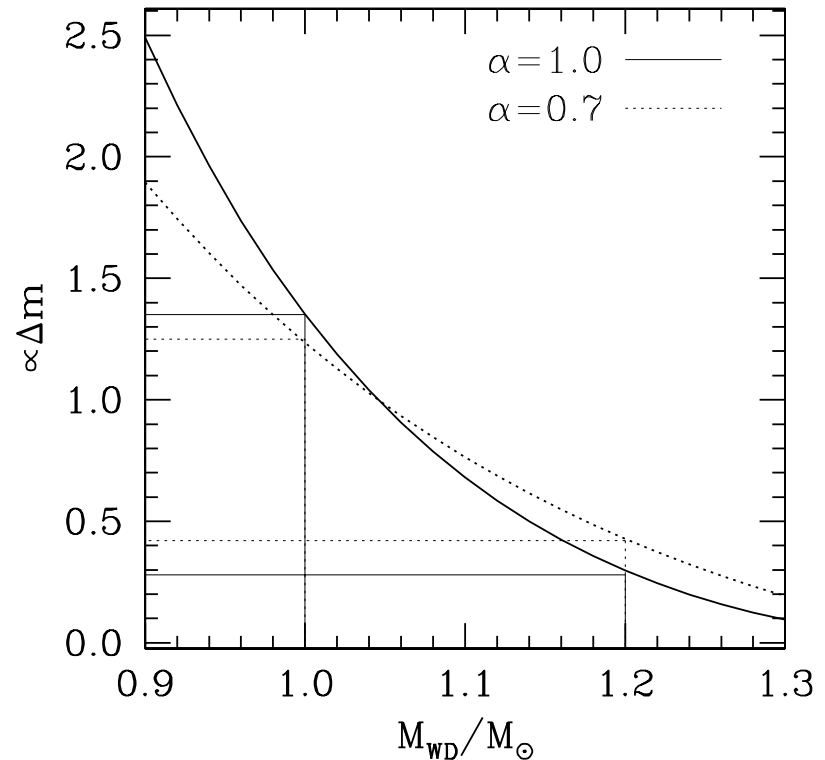

Fig. 4. Mass accreted between outbursts as a function of the white dwarf mass.

of Truran \& Livio (1986) and Ritter et al. (1991). We assume that a white dwarf undergoes a thermonuclear flash when a certain critical pressure, $P_{\text {crit }}$, is reached. A good approximation to this quantity is (MacDonald 1983):

$P_{\text {crit }}=\frac{G M_{\mathrm{WD}} \Delta m}{4 \pi R_{\mathrm{WD}}^{4}}$

where $M_{\mathrm{WD}}$ and $R_{\mathrm{WD}}$ are the white dwarf mass and radius, respectively, and $\Delta m$ is the critical mass of the accreted layer (Truran \& Livio 1986). The amount of accreted mass necessary for the ignition to take place can be obtained from a fit to full numerical simulations (Fujimoto 1982; Prialnik et al. 1982):

$\Delta m \propto\left(\frac{M_{\mathrm{WD}}}{R_{\mathrm{WD}}{ }^{4}}\right)^{-\alpha} \dot{M}_{\mathrm{WD}}^{-\beta}$

where $\alpha$ and $\beta$ are parameters to be fitted to detailed nova simulations, and $\dot{M}_{\mathrm{WD}}$ is the accretion rate. The calculations of Fujimoto (1982) and Prialnik et al. (1982) show only a weak dependence on the mass accretion rate (Ritter et al. 1991) and, therefore, we will assume $\beta=0$. This is tantamount to assuming that the recurrence time does not influence the nova distribution (Ritter et al. 1991). Note as well that calculations (MacDonald 1983), show a weak dependence on the initial luminosity, which will also be neglected here. On the other hand, $\alpha$ is typically $\simeq 1.0$ (Truran \& Livio 1986). However, numerical calculations which include a detailed treatment of diffusion (Kovetz \& Prialnik 1985) show that $\alpha=0.7$ may be a more appropriate value. Therefore, we will perform calculations for both $\alpha=1.0$ and $\alpha=0.7$.

Figure 4 shows the masses of the layers accreted between successive outbursts as a function of the mass of the white dwarf, for both choices of $\alpha$. The thin lines indicate the accreted masses for a $1.0 M_{\odot}$ and a $1.2 M_{\odot}$ white dwarf, which are, respectively, the lower mass limit allowing for the formation of an $\mathrm{ONe}$ white dwarf and a representative value of the
Table 2. Frequency of occurrence of novae of different types.

\begin{tabular}{lllllll}
\hline \hline & & \multicolumn{2}{c}{$\mathrm{SS}$} & & \multicolumn{2}{c}{$\mathrm{CBS}$} \\
\cline { 3 - 4 } \cline { 7 - 7 } & & $P_{\mathrm{CO}}$ & $P_{\mathrm{ONe}}$ & & $P_{\mathrm{CO}}$ & $P_{\mathrm{ONe}}$ \\
\hline \multirow{2}{*}{$\alpha=1.0$} & $n=0$ & 0.54 & 0.46 & & 0.68 & 0.32 \\
& $n=1 / 2$ & 0.51 & 0.49 & & 0.63 & 0.37 \\
& $n=3 / 4$ & 0.49 & 0.51 & & 0.58 & 0.42 \\
& $n=0.7$ & 0.55 & 0.45 & & 0.67 & 0.33 \\
& $n=1 / 2$ & 0.51 & 0.49 & & 0.63 & 0.37 \\
& $n=3 / 4$ & 0.50 & 0.50 & & 0.60 & 0.40 \\
\hline
\end{tabular}

mass of ONe white dwarfs. As one can see, the mass of the accreted layers changes by a factor of roughly 4.5 for this range of masses if $\alpha=1.0$ is used, whereas this change is slightly smaller (only a factor of 3 ) if $\alpha=0.7$ is adopted. If we assume that mixing is not efficient enough to allow for significant neon enrichments until most of the CO-rich layer is removed, the resulting mass of the white dwarf will be smaller when the first neon enrichments show up. Thus, more mass needs to be accreted in order to produce an outburst, as clearly seen in Fig. 4. This could possibly lead to a correlation between high neon and heavy metal enrichments and high ejected masses, as observed in some novae, but detailed calculations, which are out of the scope of this paper, should be carried out in order to check whether this is true or not.

The time interval between outbursts can be computed as the ratio between the mass accretion rate and the mass of the layer required to reach $P_{\text {crit }}$. Following Ritter et al. (1991), a selection function is defined as:

$S_{\mathrm{N}}\left(M_{\mathrm{WD}}\right)=L^{n}\left(M_{\mathrm{WD}}\right)\left(\frac{M_{\mathrm{WD}}}{R_{\mathrm{WD}}^{4}}\right)^{\alpha}$.

Here $L\left(M_{\mathrm{WD}}\right)$ is the luminosity of the hydrogen burning shell - for which we adopt the prescription of Kippenhahn (1981) and $R\left(M_{\mathrm{WD}}\right)$ is determined using the mass-radius relationship of Nauenberg (1972). The exponent $n$ in Eq. (3) accounts for the possible spatial distribution of novae (Ritter 1986). For a volume-limited sample the exponent is $n=0$; the value $n=1 / 2$ corresponds to a flux-limited sample from a disk-like distribution where interstellar extinction is taken into account; the value $n=3 / 4$ corresponds to a flux-limited sample from an isotropic distribution where interstellar extinction is taken into account (Ritter et al. 1991).

The relative probability of observing an outburst from a nova on a white dwarf which descended from a main sequence star of mass $M_{\text {ZAMS }}$ is given by

$P\left(M_{\mathrm{WD}}\right)=S_{\mathrm{N}}\left(M_{\mathrm{WD}}\right) \times M_{\mathrm{ZAMS}}{ }^{-2.35}$

where we have assumed a Salpeter IMF (Salpeter 1955). A more precise treatment of the problem should include a statistical study of the orbital parameters of the binaries leading to novae (Politano 1996) and of the effects of mass loss from the white dwarf, but for our purposes the approach adopted here is adequate. With all these inputs our results for the frequencies of occurrence of novae of the different types are shown in Table 2. Columns 3 and 4 give the occurrence frequencies for 


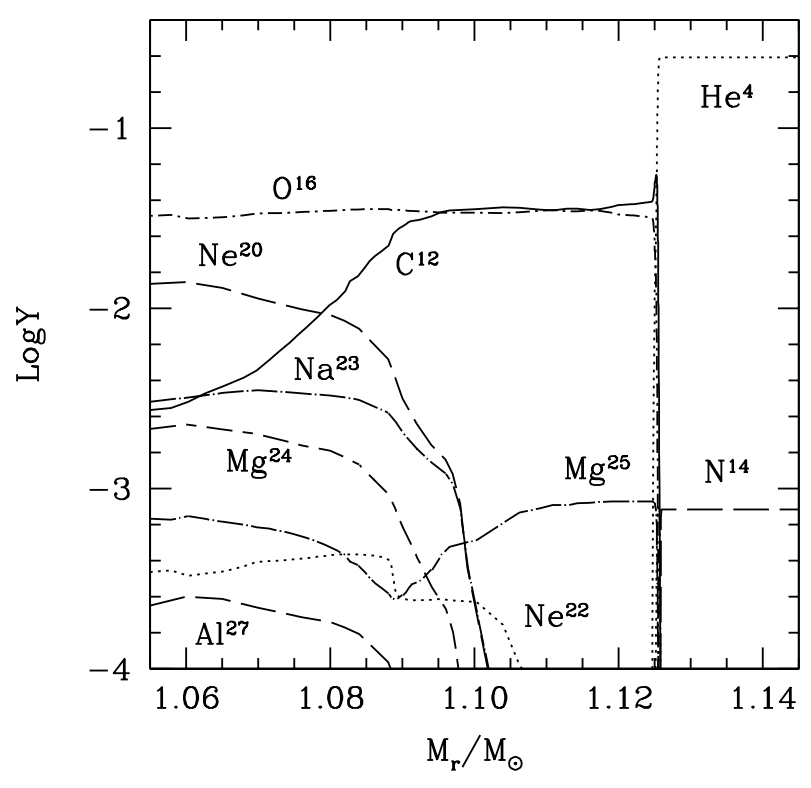

Fig. 5. Number abundances of the uppermost regions of an ONe white dwarf resulting from the evolution of a $10 M_{\odot}$ ZAMS primary component in a close binary system.

the case for which the initial-to-final mass relation obtained for the evolution of isolated stars is adopted; Cols. 5 and 6 show the results obtained for evolution in close binaries. Note that when the results for the evolution of isolated stars are adopted, the frequency of novae hosting an ONe white dwarf is always larger than that obtained for the evolution of a CBS. Thus, the effects associated with binary evolution act to reduce the fraction of $\mathrm{ONe}$ white dwarfs in nova systems relative to a single star distribution. This stems directly from the fact that the masses of the cores are considerably smaller for the evolution in a CBS.

With regard to the effects of the spatial distribution it is worth noticing that: (1) for the case of a volume-limited distribution the fraction of novae in which an ONe white dwarf is involved is roughly $1 / 3$; (2) for a disk-like distribution this fraction is slightly higher; and (3) for the case of an isotropic distribution it increases up to a value of $40 \%$. This means that the choice of a disk-like distribution, which is more appropriate to account for the distribution of Galactic novae, also increases the selection bias favouring more massive white dwarfs. This trend is even clearer for an isotropic distribution in which interstellar absorption is taken into account $(n=3 / 4)$. Finally, the effects of adopting different values for $\alpha$ (that is the effects associated to different choices of the critical pressure) are much weaker than those associated with considering different spatial distributions (different values of $n$ ).

\section{The chemical composition of the ejecta}

Figure 5 shows the profiles of the chemical abundances of the remnant of the $10 M_{\odot}$ primary component of a CBS. As discussed previously, this profile is characterized by an $\mathrm{ONe}$ rich core on top of which there is a $\mathrm{CO}$ rich buffer. The existence of this layer has consequences for the expected abundances of the nova ejecta, as the innermost material cannot be dredged-up and expelled until significant erosion of the surrounding $\mathrm{CO}$ layer has occurred, due to earlier nova outbursts. It is not easy to give a precise estimate of the number of outbursts that must occur before a significant amount of neon can be observed, since there are two factors which are not well understood and play an important role. The first factor is the mixing efficiency in the uppermost layers of the white dwarf: how fast this process operates and how much material synthesized during carbon burning is dredged up to its surface. The second factor, of a very different nature, has to do with the possibility of a variation in the recurrence time. Even though it is often assumed that the recurrence time remains constant, there are reasons to suspect that it may change.

First of all, changes in the mass transfer rate from the secondary that may occur as a consequence of the nova outbursts can modify the recurrence time. However, Kolb et al. (2001) have shown that these nova-induced mass transfer variations do not play an important role if mass transfer is sufficiently stable. There is another possibility for changes in the recurrence time, that has to do with eventual changes of the white dwarf mass. These changes are not easy to quantify but the fact that some nova abundance patterns show high metal enhancements, together with our results for the ONe white dwarf composition profiles ( $\mathrm{ONe}$ core surrounded by $\mathrm{CO}$ buffer), suggest that erosion of the surface material of the degenerate component must exist, and therefore, the white dwarf mass must decrease. The chemical composition of the surrounding layers can also modify the recurrence time in the following sense: if the amount of carbon increases, the reactions of the CNO cycle are enhanced and, as demonstrated by José \& Hernanz (1998), a smaller amount of matter needs to be accreted between outbursts in order to develop a thermonuclear runaway. Hence, the recurrence time decreases.

\subsection{The carbon-oxygen rich layer}

For comparison purposes, we present in Fig. 6 the profiles of chemical abundances at the outer shells of a $\mathrm{CO}$ white dwarf. The main isotopes are carbon and oxygen, as they were for the surface (buffer) region of the ONe white dwarf, but the different prior evolutionary histories of these remnants have left their imprints in the compositions of other isotopes. In particular, non-negligible abundances of ${ }^{20} \mathrm{Ne},{ }^{23} \mathrm{Na}$ and ${ }^{24} \mathrm{Mg}$ appear at the innermost part of the $\mathrm{CO}$ layer of an $\mathrm{ONe}$ white dwarf, whereas these elements are absent in a $\mathrm{CO}$ white dwarf. Such composition differences should be kept in mind in interpretations of the observed abundance patterns in nova nebular remnants.

It is also interesting to compare the CO-rich buffers of the remnant of the $10 M_{\odot}$ ZAMS primary (Fig. 5) with that of the corresponding single star - Fig. 9 in Ritossa et al. (1996) in order to illustrate the importance of our consideration of the effects of binary evolution. The most significant difference is in the size of the CO buffer layer, that may be seen to be thicker for the remnant of the primary in a CBS $\left(0.07 M_{\odot}\right.$ versus $0.01 M_{\odot}$ for the isolated white dwarf). Since the relative size of the ONe core is important to considerations of the 


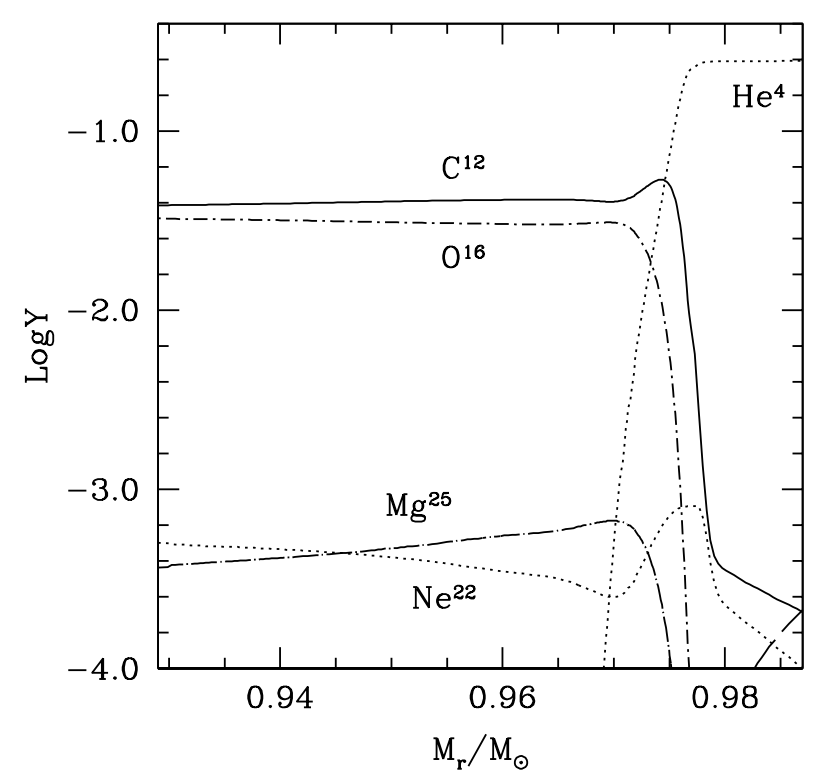

Fig. 6. Number abundances of the $\mathrm{CO}$ core resulting from a $9 M_{\odot}$ ZAMS primary component in a CBS.

relative frequencies of occurrence of $\mathrm{CO}$ versus $\mathrm{ONe}$ novae, the change of about $0.1 M_{\odot}$ is of interest. Moreover, the remnant of the binary component has a somewhat larger carbon abundance than that of the single ONe white dwarf, which can influence the runaway timescale and the size of the accreted envelope.

\subsection{The oxygen-neon cores}

The comparison of the inner ONe cores obtained from the evolution of a $10 M_{\odot}$ single star with the remnant of the $10 M_{\odot}$ primary component of a CBS shows that the final masses are slightly different $\left(1.18 M_{\odot}\right.$ and $1.06 M_{\odot}$, respectively). This implies that neon-enriched outbursts will occur in a less degenerate medium than that expected if single evolution is followed and, therefore, they will be less violent. In fact we obtain a degeneracy parameter at the bottom of the $\mathrm{CO}$ buffer which is considerably smaller $(\sim 33 \%)$ for the case of the remnant of the primary star of a CBS. Additionally, the critical mass $\Delta m$ needed to develop a thermonuclear runaway also changes. This effect can be estimated with the help of Fig. 4. As one can see, the critical mass that can be accreted by a $1.06 M_{\odot}$ white dwarf (the remnant of the CBS) is about 2.6 times larger than the critical mass that can be accreted by a $1.18 M_{\odot}$ white dwarf (the remnant of the single star). Consequently, the possibility of accreting more mass between outbursts arises. This may help us to understand an apparent observational trend that is somewhat of a challenge to theory: the fact that high neon and heavy element enrichments are sometimes found to be associated with novae with larger ejecta masses. Subsequent to the dredgeup and ejection of the overlying carbon-oxygen buffer layer, the masses of the residual ONe cores we have calculated allow the possibility of accreted envelopes approaching $10^{-4} M_{\odot}$. Moreover, if the remaining parameters of interest are kept constant, the recurrence time between outbursts will change as the mass of the critical accreted layer and, therefore, the frequency in this case will decrease by roughly a factor of 2.0-2.6.

\section{Discussion and conclusions}

The calculations described in this paper may be summarized as follows:

- We have computed self-consistent evolutionary sequences for the formation of white dwarfs with ONe cores in close binary systems involving intermediate mass stars. We have found that the minimum mass at the ZAMS which allows for extensive carbon burning in the $\mathrm{CO}$ core is $\simeq 9.3 M_{\odot}$, and that the mass of the resulting white dwarf is, in this case, $M_{\mathrm{WD}} \simeq 1.1 M_{\odot}$. This value is somewhat lower than previous limits on the masses of ONe white dwarfs.

- We have obtained the initial-to-final mass relationship and used our evolutionary sequences to revisit the question of the relative fraction of novae which contain white dwarfs with $\mathrm{ONe}$ cores. Our motivation to reconsider this problem has been the major influence of the white dwarf mass (through the mass-radius relation) on the selection effects that favour the observation of outbursts produced in more massive novae. In our determination of the relative frequency of novae with ONe cores, we have followed closely the treatments of Truran \& Livio (1986) and Ritter et al. (1991).

- We have found that the primary effect of binarity consists of a noticeable reduction in the frequency of novae hosting ONe white dwarfs. Specifically, when we take into account our results for evolution in a CBS, we find that approximately $37 \%$ of the observed nova outbursts are expected to host $\mathrm{ONe}$ white dwarfs for a disk-like distribution. This fraction decreases to $32 \%$ for a volume-limited sample. Since the Galactic distribution of classical novae extends beyond the thickness of the Galactic disk and interstellar absorption plays an important role, the relevant distribution is therefore given by a disk-like distribution - that is, $n=1 / 2$ in Eq. (3). These results imply that flux limitation favours observation of the most luminous novae: those occurring on the most massive white dwarfs.

- We have also calculated detailed composition profiles for these remnants. This is one of our major results. We have found that the remnants of the evolution in CBS always have a relatively thick $\mathrm{CO}$ buffer on top of the $\mathrm{ONe}$ core. This means that novae hosting massive white dwarfs with $\mathrm{ONe}$ cores can show $\mathrm{Ne}$ enrichments only if either the $\mathrm{CO}$ buffer has been removed by successive nova outbursts or been mixed with material from the ONe core by some other mechanism. In contrast, if the mass transfer process has started only recently, the thermonuclear runaway will proceed on top of the $\mathrm{CO}$ buffer and the ejecta will reflect the abundance pattern of a massive $\mathrm{CO}$ nova. Of course, ultimately the $\mathrm{CO}$ surface layer will be eroded and the ONe core will be exposed.

- As pointed out by Livio \& Truran (1994), the interpretation of the observed yields of novae remains somewhat uncertain. Stellar evolution theory clearly predicts that, at birth, $\mathrm{ONe}$ white dwarfs are more massive than $\mathrm{CO}$ white dwarfs. However, the rather massive nebular remnants inferred for some novae with large neon enrichments are difficult to reproduce on the basis of current theoretical nova 
simulations. Our models seem to give some hints towards a (partial) solution to this problem. First, we have pointed out that ONe white dwarfs do not have a homogeneous composition profile but, rather, consist of two clearly different regions. The dominant elements in the inner zone are oxygen and neon, while the overlying layer is mainly composed of carbon and oxygen. This $\mathrm{CO}$ buffer turns out to be important when interpreting the nature of the nova ejecta, since its size is not the same for all ONe white dwarfs: it is smaller for more massive white dwarfs (see Table 1). When the CO buffer is expelled after a series of outbursts, we are left with a naked ONe white dwarf, whose typical mass is between approximately 1.0 and $1.1 M_{\odot}$ but ranges up to the Chandrasekhar limit. For the lowest mass cases, the white dwarf cores can even be somewhat less massive than the most massive $\mathrm{CO}$ white dwarfs, and thus can be expected to accrete rather massive envelopes before the thermonuclear runaway ensues. This may help to explain the rather large masses that have been quoted for the ejecta of such identified "neon novae" as V1370 Aql 1982 and QU Vul 1984 (Saizar et al. 1992; Andreä et al. 1994; Schwarz 2002).

It seems clear that the last word on these questions has not yet been told, but we believe that our results for ONe white dwarfs resulting from close binary evolution will help to provide a solid basis for a better understanding of nova explosions.

Acknowledgements. Part of this work was supported by the Spanish DGES project number PB98-1183-C03-02/03, by the MCYT grants AYA2000-1785, AYA2001-2360, and by the CIRIT. JWT would like to acknowledge support by the US Department of Energy under Grants B523820 and DE-FG02-91ER40606 at the University of Chicago. We also would like to acknowledge our referee, H. Ritter, for his very valuable suggestions.

\section{References}

Andreä, J., Drechsel, H., \& Starrfield, S. 1994, A\&A, 291, 869 Austin, S. J., Wagner, R. M., Starrfield, S., et al. 1996, AJ, 111, 869 Bergeron, P., Saffer, R. A., \& Liebert, J. 1992, ApJ, 394, 228

Calder, A. C., Alexakis, A., Dursi, L. J., et al. 2002, in Classical nova explosions, ed. M. Hernanz, \& J. José, AIP Conf. Proc., 637, 134

Delgado, A. J., \& Thomas, H.-C. 1981, A\&A, 96, 142

Fujimoto, M. Y. 1982, ApJ, 257, 767

Gehrz, R. D., Truran, J. W., Williams, R. E., \& Starrfield, S. 1998, PASP, 110,3

Gil-Pons, P., \& García-Berro, E. 2001, A\&A, 375, 87
Gil-Pons, P., \& García-Berro, E. 2002, A\&A, 396, 589

Glasner, S. A., Livne, E., \& Truran, J. W. 1997, ApJ, 475, 754

Iben, I., Jr. 1990, in Confrontation between stellar pulsation and evolution, ed. C. Cacciari, \& G. Clementini, ASP Conf. Proc., 11, 483

Iben, I., Jr., \& Tutukov, A. 1985, ApJS, 58, 661

José, J., \& Hernanz, M. 1998, ApJ, 494, 680

José, J., Hernanz, M., \& García-Berro, E. 2003, Nucl. Phys. A, 718, 255

Kippenhahn, R. 1981, A\&A, 102, 293

Kippenhahn, R., \& Thomas, H. C. 1978, A\&A, 63, 265

Kolb, U., Rappaport, S., Schenker, K., \& Howell, S. 2001, ApJ, 563, 958

Kovetz, A., \& Prialnik, D. 1985, ApJ, 291, 812

Law, W. Y., \& Ritter, H. 1983, A\&A, 123, 33

Livio, M. 1992, in Viña del Mar Workshop on Cataclismic Variable Stars, ed. N. Vogt, ASP Conf. Ser., 29, 4

Livio, M., \& Truran, J. W. 1994, ApJ, 425, 797

Livio, M., \& Truran, J. W. 1987, ApJ, 318, 316

Livio, M., \& Soker, N. 1984, MNRAS, 208, 783

de Loore, C., \& Doom, C. W. H. 1992, Structure and evolution of single and binary stars, Astrophysics and Space Science Library, vol. 179 (Dordrecht: Kluwer)

MacDonald, J. 1983, ApJ, 267, 732

Nauenberg, M. 1972, ApJ, 175, 417

Paczynski, B. 1976, Structure and Evolution of Close Binary Systems, ed. P. P. Eggleton, S. Mitton, \& J. Whelan (Dordrecht: Reidel), 75

Politano, M. 2002, in Classical nova explosions, ed. M. Hernanz, \& J. José, AIP Conf. Proc., 637, 409

Politano, M. 1996, ApJ, 465, 338

Prialnik, D., Livio, M., Shaviv, G., \& Kovetz, A. 1982, ApJ, 257, 312

Prialnik, D., \& Kovetz, A. 1984, ApJ, 281, 376

Ritter, H. 1986, A\&A, 168, 105

Ritter, H., \& Burkert, A. 1986, A\&A, 158, 161

Ritter, H., Politano, M., Livio, M., \& Webbink, R. F. 1991, ApJ, 376, 177

Ritossa, C., García-Berro, E., \& Iben, I., Jr. 1996, ApJ, 460, 489

Rosner, R., Alexakis, A., Young, Y.-N., Truran, J. W., \& Hillebrandt, W. 2001, ApJ, 562, L177

Saizar, P., Starrfield, S., Ferland, G. J., et al. 1992, ApJ, 398, 651

Salpeter, E. E. 1955, ApJ, 121, 161

Schwarz, G. J. 2002, ApJ, 557, 950

Shara, M. M. 1994, AJ, 107, 1546

Shara, M. M., \& Prialnik, D. 1994, AJ, 107, 1542

Truran, J. W., \& Livio, M. 1986, ApJ, 308, 721

Vanlandingham, K. M., Starrfield, S., \& Shore, S. N. 1997, MNRAS, 290, 87

Webbink, R. F. 1990, in The Physics of Classical Novae, ed. A. Cassatella, \& R. Viotti (Berlin: Springer), Proc. IAU Coll., 122, 405 